\title{
Natural risk assessment and decision planning for disaster mitigation
}

\author{
P. Fiorucci, F. Gaetani, R. Minciardi, and E. Trasforini \\ CIMA Centro di ricerca Interuniversitario in Monitoraggio Ambientale, Savona, Italy \\ Received: 30 November 2004 - Revised: 14 April 2005 - Accepted: 18 April 2005 - Published: 13 May 2005
}

\begin{abstract}
In this paper, decisional models are introduced aiming at defining a general framework for natural disaster mitigation. More specifically, an integrated approach based on system modelling and optimal resource assignment is presented in order to support the decision makers in preoperational and real-time management of forest fire emergencies. Some strategies for pre-operative and real time risk management will be described and formalized as optimal resource assignment problems. To this end, some models capable to describe the resources dynamics will be introduced, both in pre-operative phase and in real-time phase.
\end{abstract}

\section{Introduction}

Among the actions that can be carried out in order to mitigate the effects of natural hazards on human lives and activities, or more generally over a territorial system, a correct management of the resources assigned to cope with such phenomena plays a very important role. This work focuses on the decisions planning phase and on the control actions that can be carried out in order to optimally manage the available resources. More specifically, the pre-operational and the real-time management phases are taken into account. In the first phase, the main objective is that of moving the resources closer to sites with a higher hazard; an appropriate model able to forecast the risk assessment identifies such sites. Instead, in the second phase, the task to achieve is that of moving the resources where they are demanded. Both problems can be formalized as mathematical programming problems.

In this paper, an integrated approach based on system modelling and optimal resource assignment is presented, in order to support the decision maker in pre-operational and real-time management of forest fire emergencies. Such an approach is briefly described in Sect. 2. In Sect. 3 the decisional models for disaster mitigation in case of forest fire

Correspondence to: P. Fiorucci

(paolo@cima.unige.it) emergencies are introduced, both for pre-operational and real-time phases.

In pre-operational phase, indications about the preventive allocations of resources (water bombers and helitankers) to face possible fire emergencies will be provided; whereas, in the real time phase, the decisions are relevant to the (dynamic) management of resources, taking into account the potential danger of each existing fire, travel times of resources, and fire suppression time. Strategies for real time risk management will be introduced, by setting an optimal control problem, in which the dynamics of the various components of the system are properly modelled. Finally some conclusion and remark will end the paper.

\section{A general framework for forest fire risk management}

As happens in connection with other kinds of natural risks, forest fire hazard assessment can take place starting within three different conceptual frameworks: static risk assessment, dynamic risk assessment and real time risk assessment. In the first one, the evaluation and the risk distribution over the territory is carried out on the basis of static information (topography, vegetational cover) and taking into account the historical series of the main variables involved in the considered process (i.e., fire occurrence, weather conditions and land use). The purpose of such an assessment could be that of planning the sizing and the location of the different kinds of resources (men, trucks, engines, aircrafts, infrastructures, etc.) necessary to manage forest fire risk over a wide territory. Within the second framework, which will be denoted as dynamic risk assessment, it is assumed that real-time information is available, and that the risk assessment is carried out with reference to a certain time horizon (say 2-3 days) for which reliable meteorological forecasts are available. Along with forecast information, the real-time information used for dynamic risk assessment may come from different sources: present weather conditions, ground-measured data relevant to vegetational conditions, and data coming 
from satellite sensors (i.e., Normalized Difference Vegetation Index, Greenness index, Leaf Area Index). Ground sensors can provide real-time information about fuel temperature and fuel moisture conditions for different fuel size (diameter) classes. In addition, soil water content sensors can provide information related with dead and live fuel moisture conditions. The satellite data are mainly used for evaluating the percentage of fuel load $\left[\mathrm{kg} / \mathrm{m}^{2}\right]$ attributable to dead or to live vegetation. Such an evaluation can be obtained on the basis of the Relative Greenness (RG) index, which represents how green each pixel is, in relation to the range of historical NDVI observations available for it.

The main advantage of dynamic risk assessment is that of identifying, within the considered territory, the areas affected by the highest risk, and the time intervals within the considered time horizon in which this risk takes place. The purpose of dynamic risk assessment is that of getting reliable information useful to take a number and a variety of preoperational actions that can reduce the impact of potentially lighted fire over the considered territory, within the considered time horizon. Such actions may include, for instance, re-locating the available resources over the territory, recalling day-off resources to service, alerting local authorities, issuing prohibitions of some dangerous agricultural practices (such as stubble burning), or patrolling the areas affected by the highest hazard.

Finally, the third framework is that corresponding to a situation in which some active fires have been detected, and the problem that has to be solved is that of selecting the best actions to fight such fires, taking into account the information corresponding to the risk distribution over the considered territory. In this case, a risk assessment relevant to each detected fire has to be carried out. On this basis, an operational decision procedure can be applied, in order to support the decision makers in taking decisions about the actions to undertake in order to contrast effectively and possibly to extinguish the detected fires.

Summing up, for any of the above-mentioned three frameworks, an information-processing phase, aiming at performing a risk assessment, has to be considered, followed by a decision-oriented phase, whose objective is the selection of the best actions to undertake, on the basis of the available information relevant to vulnerability and costs of the exposed elements.

What distinguishes the three frameworks, which can be denoted as information processing/decision levels, is the time horizon characterizing their operationality and the available information. Clearly, information generated at any of the levels is passed to the following ones, as it shown in Fig. 1.

This paper is focused on the second and the third framework. In particular, dynamic risk assessment information is relevant to the outputs of a model in use by the Italian Civil Protection (Fiorucci et al., 2004), whereas information relevant to active fires is provided by a semi-physical propagation model (Rothermel, 1972). In the next section, the decisional models, based on the information introduced above, are formalized and briefly described.

\section{Decision models}

In the next subsections, the formalizations of the preoperational and the real-time resource allocation problems for forest fire risk management will be provided. Both the problems are formalized as mathematical programming problems. Even if optimal resource allocation is one of the most widely studied areas in mathematical programming (Kozanidis and Melachrinoudis, 2004), only few works addressing the optimal resource allocation for emergency management in connection to natural hazards can be found in literature; among them see the work of Friedrich et al. (2000), who propose the formulation of an optimisation problem for resource allocation for emergency response after earthquake disasters, and Paggio et al. (1999), who present, in the resource management module of the TRACE system, an allocation procedure aiming at defining a resource allocation plan.

In this work, for the sake of brevity only a summarized treatment is provided, since only the cost functions of the two optimisation problems will be analysed in details, whereas the constraints of the problem will be only shortly discussed.

\subsection{Pre-operational resource management}

The information related to the expected daily forest fire risk is used as input for a pre-operational resource allocation problem at a national scale, on the basis of the assumption that the timelier is the intervention, the more efficient is the action of a resource on a fire. The area of study (i.e., the Italian territory) is represented as a discretised spatial domain, defined by a grid of $K$ regular cells; within each cell $k$, the demand is supposed to be homogeneous, and it is represented through the use of parameter $D_{k}$. The dimension of such cells is that of the ones characterising the dynamic risk assessment model, namely $0.05^{\circ}$ side length. It has to be underlined that the resources are not actually positioned in cell $k$, as they are assigned to the location centres $j(j \in V$, where $V$ is the set of location centres) spread over the territory. Such centres are assumed to be the airbases able to receive the resources, and able to provide the service of maintenance needed to ensure the effectiveness of the aircraft for the next flights. The location centres can be treated as nodes of a graph, superimposed to the grid. In this way, the resources have to traverse the links of the graph in order to reach the nodes to which they are assigned.

The binary decision variable of the problem is $x_{i j}$, and it is defined for each resource $i(i=1, \ldots, R)$ and for each resource location centre $j(j \in V)$; such a variable assumes value equal to 1 when the resource is assigned to the resource location centre, and 0 otherwise.

Further on, some parameters have to be introduced. At first, the cost $\phi_{i j}$ relevant to the transfer of resource $i$ from the current position to node $j$ has to be taken into account, as well as the degree of closeness of cell $k$ to the nearest water supply point $\sigma_{k}$, and the degree of easiness of reaching cell $k$ by resources located at location centre $j$, namely $\rho_{j k}$. 


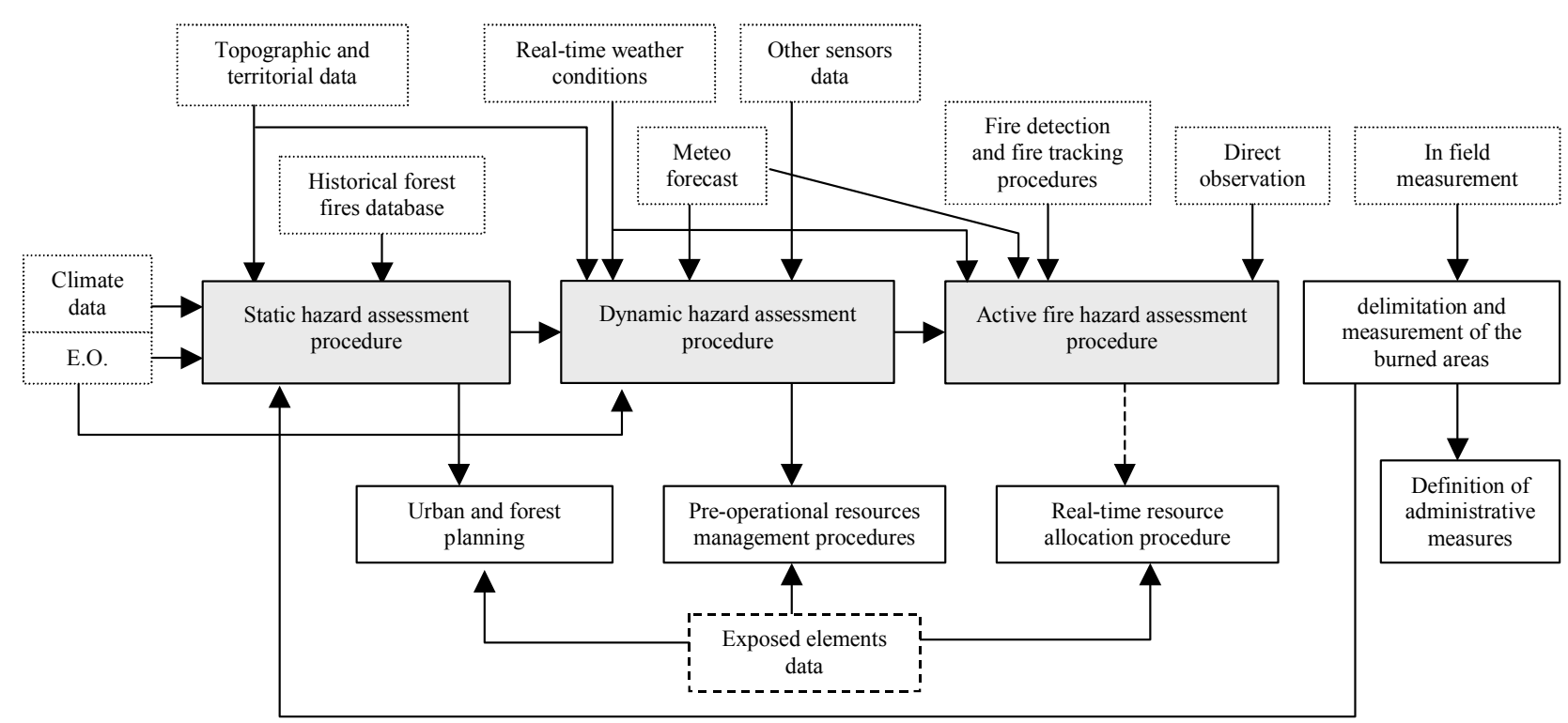

Fig. 1. A schematic representation of the various functions and of the information flows in an overall forest fire management scheme.

Indeed, when a pre-operational location problem has to be set up, the decision to reallocate an aircraft from an airport to another, one presupposes high operative cost. Therefore, such a (hard) decision has to be taken only when a very high and persistent hazard is forecasted on a geographical area, and not only on the basis of extreme and isolated hazard values. For such a reason, decisions in the pre-operational location problem will be based on considerations relevant to both the necessity to suitable cover all the considered area, and the advisability to move the resources from their actual position. Thus, the cost function of the optimisation problem is composed of two terms. The first term is relevant to the unsatisfied service demand in each area that can be reached by a resource assigned to a specific location, whereas the second term is related to the cost relevant to the actual transfer of a resource from the current position to location centre. The cost function of the pre-operational resource allocation problem can be formalized as follows

$$
\begin{aligned}
& \min Z=\sum_{j \in V} \max \left(\sum_{k=1}^{K} D_{k} \sigma_{k} \rho_{j k}-\delta \sum_{i=1}^{R} x_{i j}, 0\right)^{2} \\
& +\alpha \sum_{j \in V} \sum_{i=1}^{R} \phi_{i j} x_{i j}
\end{aligned}
$$

where $\alpha$ is a suitable weighting parameter, aiming to balance the two terms of the cost function. Referring to the first of such terms, parameter $\delta$ is a suitable trade-off parameter, introduced in order to make the two terms in the "max" function comparable. Such a parameter depends on the technical characteristics of the considered resources, as it can be viewed as a measure of the capability of the resources to cope with several fires in the same time period.

The first term of the cost function is quadratic as it seems sensible to penalize at most the unsatisfied demand related to those cells characterized by a high value of service demand. Moreover, the cost function is non-linear because of the introduction of the "max" function. Nevertheless, such a function can be transformed into a quadratic one by the use of well-known devices of the operations research. The description of such devices is out of the scopes of this paper, but one can find an explanation of some of theme in, among the others, Haith (1982).

It has to be noted that the service demand (weighted by parameters $\sigma_{k}$ and $\rho_{j k}$ ) is aggregated in the cost function over the influence area of each location centre, and thus it could be sufficient to evaluate off-line such values for each location centre. Nevertheless, it was chosen to propose the formulation in Eq. (1) in order to underline the importance of parameters $\sigma_{k}$ and $\rho_{j k}$ Otherwise, an interesting improvement of the proposed work should be the sensitivity analysis of the obtained results with respect to the cell dimension (and thus of the spatial representation of parameters $D_{k}, \sigma_{k}$ and $\rho_{j k}$ ).

Some constraints are to be introduced in order to obtain suitable indications for the forest fire emergencies preoperational resource allocation problem. More specifically, a constraint stating that the each resource are to be assigned to one and only one resource location centre, and a constraint stating that the decisional variables $x_{i j}$ are binary have to be taken into account.

The result of such a decision model is the allocation of the aerial resources on the national Italian territory for the next day, namely the value of variable $x_{i j}$ in the optimal solution of the problem for each resource $i$, and for each location centre $j$. Figure 2 shows an example of the results of the application of the proposed approach to the Italian territory. The black numbers indicate the number of aerial resources assigned to each airport. Different colours indicate instead different levels of coverage of the considered area by the aerial 


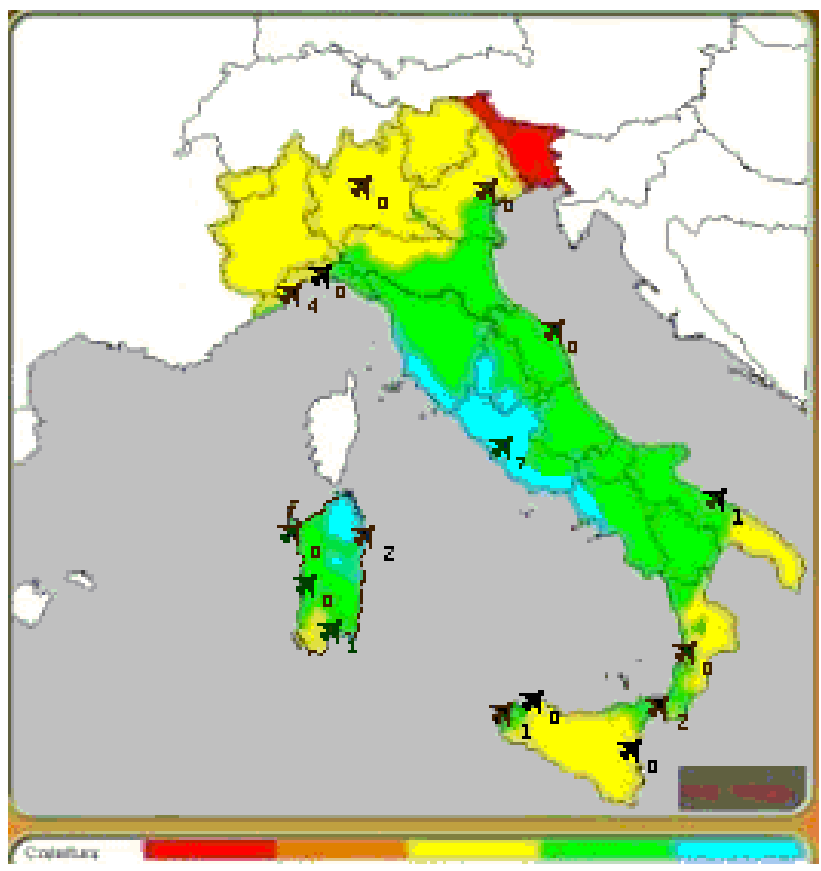

Fig. 2. An example of results obtained following the approach proposed in Sect. 3.1. The airplanes represented in pictograms represent the 16 national airbases considered by the procedure, whereas the numbers near the pictograms indicate the amount of resources relocate to each airports. A palette of colour has been used in order to colour the Italian territory in connection to the coverage assured by the resource relocation. Red areas represent the least covered ones, whereas blue areas are the more covered by available resources.

means, taking into account the closeness to the nearest water supply point, and the degree of easiness of reaching the area by the location centre where the resource are actually assigned.

\subsection{Real-time resource allocation}

In this problem, stated and solved at local scale, it is assumed that both demand centres and resource location centres are represented as nodes of the directed graph $\mathrm{G}(V, L)$, where $V$ is again the set of nodes, and $L$ is the set of the links among those nodes. The general problem formalization refers to a time horizon (of a suitable length) of $T$ time intervals. It is assumed that each link belonging to the set $L$ is characterized by a unitary transit time, that is the time required by the resources in order to transit over the link. This assumption can be generalized to include links with transit time greater than one, by introducing a suitable number of dummy nodes, each one characterized by null service demand.

In this case, two are the primary decision variables, i.e. the variables whose values can be modified by the end users in order to obtain a specific objective. More specifically, they are the amount $U_{j}(t)$ of resources assigned to node $j$ during time interval $(t, t+1)$, and the amount $w_{j l}(t)$ of resources that during time interval $(t, t+1)$, move from node $j$ to node $l$.
Then, two other variables are used in order to describe the state of the system (i.e., the fire), namely the cumulated burnt area $a_{j}(t)$ relevant to the fire $j$ in time interval $(t, t+1)$, and the power $p_{j}(t)$ of fire $j$ in time interval $(t, t+1)$. Note that, in this case, the service demand can be assimilated to the second of such variables, as the work of the resources aims to extinguish the fires, i.e. to annul the power of such a fire. The cumulated burnt area is instead a measure of the damage induced by the fire, so a choice concordant to the need of minimizing the effect of wild fires on the environment is that of inserting such a variable in the cost function of the optimisation problem.

Another term can be inserted into the cost function, namely the transfer cost. Such a cost is introduced in order to penalize the resource movements among the nodes, and it takes into account the total amount of resources moving on each link for each time interval. With this assumption, the cost function of the real-time resource allocation problem can be defined as follows

$$
\min \sum_{t=0}^{T-1} \sum_{j \in Y} \gamma_{j, t} a_{j}^{2}(t)+\sum_{t=0}^{T-1} \sum_{j \in V} \sum_{\substack{l \in V \\ l \neq j}} \eta_{j, l, t} w_{j l}^{2}(t)
$$

where $\gamma_{j, t}$ and $\eta_{j, l, t}$ are suitable weight parameters, and where $Y$ is a sub-set of set $V$ constituted by the active fires.

As a matter of fact, natural hazard management is in general characterized by two different dynamics: the first one is relevant to the physical process that characterizes the specific event (flood, forest fires, etc.), whereas the second one is relevant to the resources intervention. In case of forest fire, risk is related with the fire spread dynamics, which is strongly correlated with the fire attack provided by the resources able to face the propagation of the fire. Thus some constraints relevant to the dynamics of both the forest fire event and of the resources have to be introduced in the formalization of the optimisation problem. The dynamics of the event can be, for instance, inserted as constraints through the use of a fire spread model (Rothermel, 1972), taking also into account the extinguishing action of the amount of resources actually assigned to such a fire during time interval $(t, t+1)$, namely $U_{j}(t)$. Furthermore, some constraints relevant to the resource dynamics over the graph, and some other imposing the non-negativity of the variables have to be taken into account.

It is important to underline that the definition of the constraint relevant to the fire dynamics deeply influences the complexity of the problem and the computational time required in order to obtain solutions. Thus, as the goal of this tool is that of helping the end users in the real time decision making, it could be preferable to use roughly simplified method allowing short computational time, rather than sophisticated method, requiring a solving time that could be not compatible with the needs of the stakeholders. 


\section{Conclusions}

A general framework for the forest fire risk management in the pre-operational and in the real-time phases has been presented and discussed. In both cases, the overall procedure is composed by two modules: a knowledge-oriented module, whose main goal is that of assessing the forest fire risk, and a decision-oriented module, aiming at optimising the resource allocation over the considered territory.

In the pre-operational phase, the information relevant to the dynamic forest fire risk is considered as an input of the optimisation problem. The goal of the problem is that of optimally assigning the resources to the considered location centres (airports) spread over the Italian national territory. The decisional variables of such a problem are the number of aerial resources assigned to each airport over the considered area. The objective of the optimisation problem is that of allocating the resources in order to minimize the number of cells denoted by high risk and not reachable by any aerial resource, taking into account the resources transfer costs.

Referring instead to the real-time phase, it has to be noted that, in this case, the risk assessment is no more a simple input of the optimisation problem, but the two modules are strictly embedded. More specifically, the service demand relevant to the forest fire risk is, differently from the previous case, not a parameter of the problem, but a variable introduced in order to characterise the state of the considered system (i.e., the fire front propagation in space and time). Besides, it is worth observing that the value of such a variable depends also on the resource intervention upon the considered fire. The cost function of such a problem, which is usually defined and solved at local scale, is given by the minimisation of the burnt area taking into account the resource transfer costs.

Further research in this field should be directed to model calibration and validation, and to the development of efficient procedures for the solution of the above problems over large regions. Besides, the modelling of a real-time resource allocation problem whose transfer times and direct/indirect costs of resource cannot be neglected, is necessary. Finally, the proposed techniques are presently under test in connection with case study in some Italian regions.
Acknowledgements. The activities reported in the paper are presently being carried out in the framework of Italian Civil Protection case study and are funded by the Gruppo Nazionale per la Difesa dalle catastrofi Idrogeologiche GNDCI, U.O. n. 3.28, Special project n. 4 (2002) Structural and operational design of a decision support system based on a national geographical information system and aiming at forest fire risk management.

Edited by: L. Ferraris

Reviewed by: anonymous referees

\section{References}

Haith, D. A.: Environmental systems optimisation, John Wiley \& Sons, USA, 1982.

Fiorucci, P., Gaetani, F., and Minciardi, R.: An integrated system for the forest fires dynamic hazard assessment over a wide area, in: Complexity and Integrated Resources Management, edited by: Pahl-Wostl, C., Schmidt, S., Rizzoli, A. E., and Jakeman, A. J., Transactions of the 2nd Biennial Meeting of the International Environmental Modelling and Software Society, ISBN 88900787-1-5, iEMSs: Manno, Switzerland, 2004.

Friedrich, F., Gehbauer, F., and Rickers, U.: Optimized resource allocation for emergency response after earthquake disasters, Safety Science 35, 2000.

Kozanidis, G. and Melachrinouids, E.: A branch and bound algorithm for the 0-1 mixed integer knapsack problem with linear multiple choice constraints, Computers and Operation Research, 31, 2004.

Paggio, R., Agre, G., Dichev, C., Umann, G., Rozman, T., Batachia, L., and Stocchero, M.: A cost-effective programmable environment for developing environmental decision support systems, Environmental Modelling and Software, 14, 1999.

Rothermel, R. C.: A mathematical model for predicting fire spread in wildland fuels, USDA, Forest Service Research Paper, INT114, Intermountain Forest and Range Experiment Station, Ogden, UT, 1972 